\title{
Circulating Cancer Cells with Relative Expression of miRNA and Cytotoxic T-cell Activities in Non-small cell Lung Cancer Patients
}

\author{
Shymaa Morad \\ Zoology Department, Tanta University, Tanta, Egypt
}

\section{III}

Background: Lung cancer is the leading cause of cancer-related death worldwide with a substantially low survival rate. MiRNAs have been shown to regulate self-renewal and differentiation properties of CSCs, Here, MicroRNAs play a significant role in shaping immune response. Aims: the current study aims to analyze the numbers of helper CD4+ and cytotoxic CD8+ T lymphocytes, CD133+ and Epcam+ CSCs. also, measure the relative expression of miRNAs in Non-small cell lung cancer patients before and after treatment. Materials and Methods: The frequencies of Epcam+ and CD133+ cells and the numbers of helper CD4+ and cytotoxic CD8+ T lymphocytes were analyzed in the peripheral blood of NSCLC patients before $(n=10)$ and after $(n=10)$ chemotherapy using multiparametric flow cytometry. The gene expression of miRNAs was measured using microarray.Results: Early diagnosed patients before treatment showed high numbers of Epcam + and CD113+ CSCs when compared to CTRL; where the numbers of these cells were decreased after treatment as compared to early diagnosed patients. A significant upregulation of miRNAs and downregulation of miRNAs were observed in the peripheral blood of NSCLC patients as compared to healthy control. The relative and absolute numbers of CD4+ and CD8+ T-cells were significantly elevated in the peripheral blood of NSCLC patients. Conclusion: This study opens a new avenue to investigate the mechanism mediating the emergence of these cells on larger number of NSCLC patients at different treatment stages. We also discuss the role of microRNAs in therapeutic resistance and as biomarkers.

Keywords: Non-small cell lung cancer (NSCLC); miro-RNA; Cancer stem cells (CSCs)

Editor-in-Chief: Prof. M.L. Salem, PhD - Article DOI: 1010.21608/jcbr.2021.61161.1162 